\title{
Preparation of novel potassium, lanthanum-iron oxides and their magnetic properties
}

\author{
Tatsuhiro Yamamoto, Kenji Kamishima, Koichi Kakizaki and Nobuyuki Hiratsuka \\ Graduate School of Science and Engineering, Saitama University, 255 Shimo-okubo, Saitama 338-8570, Japan \\ Fax: 048-858-9131, e-mail: kamisima@fms.saitama-u.ac.jp
}

\begin{abstract}
We have investigated the synthesis conditions, and the magnetic properties of M-type ferromagnetic potassium, lanthanum-iron oxides. The samples were prepared by solid-state-reaction. It is found that a new M-type ferrite can be effectively synthesized at $1275^{\circ} \mathrm{C}$ from the starting materials pulverized with a planetary ball mill. The magnetic transition temperature of the sample is $440^{\circ} \mathrm{C}$ which is quite close to that of the magnetoplumbite-type ferrite. EPMA compositional analysis for this new M-type ferrite shows the composition ratio of $\mathrm{K}: \mathrm{La}: \mathrm{Fe}=0.75: 0.90: 18.58$. From this result, the chemical formula of the magnetoplumbite phase in this sample is estimated to be $\mathrm{K}_{0.45} \mathrm{La}_{0.55} \mathrm{Fe}_{12} \mathrm{O}_{19-s \text {. }}$
\end{abstract}

Key words: potassium, lanthanum, magnetoplumbite, $\mathrm{LaFe}_{12} \mathrm{O}_{19}$

\section{INTRODUCTION}

The hexagonal ferrites, $\mathrm{MFe}_{12} \mathrm{O}_{19}(\mathrm{M}=\mathrm{Ba}, \mathrm{Sr}, \mathrm{Pb})$ with the magnetoplumbite structure are well-known ferromagnetic iron-oxides for ceramic permanent magnetic materials at present. They have high saturation magnetization, high resistance, high chemical stability and high Curie temperatures. Their coercive field strength can be adjusted for a wide range of applications [1]. A basic feature of hexagonal magnetoplumbite structures is a layer of relatively large $\mathrm{O}^{2-}$ and $\mathrm{M}^{2+}$ ions arranged essentially in close-packed fashion, that is, each large ion is surrounded by six other large ions in the plane. The close-packed layers form two kinds of blocks, one is a spinel block and the other is an R block as shown in Fig. 1. In the spinel block, a transition metal cation is located at an octahedral site with six oxygen ligands or at a tetrahedral site with four oxygen ligands. Another close-packed large ion layer has a $\mathrm{Ba}^{2+}$ ion substituted for every fourth $\mathrm{O}^{2-}$ ion, which gives an ordered 1:3 ratio of alkaline earth metal cation to oxygen anion. When this Ba-O type layer is sandwiched between two oxygen layers, the total three layers become an R block. A transition metal cation just on the site with five oxygen ligands, which produce a uniaxial ligand field. Other sites between the Ba-O type and oxygen layers in the $\mathrm{R}$ block are octahedral. The hard magnetic properties of the M-type ferrites come from this magnetocrystalline anisotropy of $\mathrm{Fe}^{3+}$ at the $\mathrm{R}$ block in the magnetoplumbite structure.

Because of their significance for industrial applications, several forms of substitutions in hexagonal ferrites have been tried with the aim to improve hard magnetic properties [2-6]. Some experiments used rare-earth ions such as $\mathrm{La}$ substitution for $\mathrm{Ba}, \mathrm{Sr}$ or $\mathrm{Pb}[7-9]$.

In the 1970's Lotgering [10] reported a remarkable increase of anisotropy $\mathrm{LaFe}_{12} \mathrm{O}_{19}$ which contains $\mathrm{Fe}^{2+}$ ions. Few investigations of this compound, however, have been carried out due to difficulties in preparing this iron-oxide. Kupferling et al. set out that $\mathrm{LaFe}_{12} \mathrm{O}_{19}$ was prepared by three different methods (mechanical

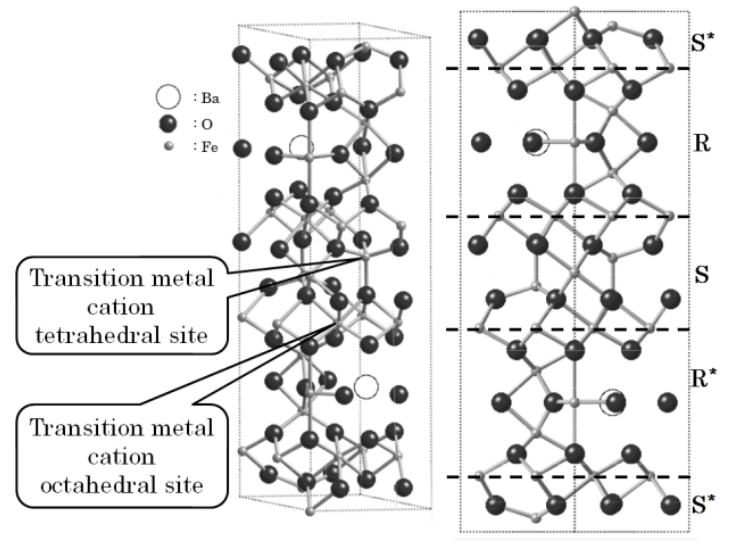

Fig. 1 Crystal structure of M-type ferrites. An asterisk denotes that the block is turned for $180^{\circ}$ around c-axis.[11]

alloying, coprecipitation and sol-gel) [12]. Since La-hexaferrite phase is only stable between 1360 and $1390^{\circ} \mathrm{C}$, in order to form this phase, a heat treatment within this temperature range and then a rapid quenching to room temperature were performed for these three samples. Nevertheless, these specimens contained $\mathrm{LaFeO}_{3}$ and $\alpha-\mathrm{Fe}_{2} \mathrm{O}_{3}$ phases together with $\mathrm{LaFe}_{12} \mathrm{O}_{19}$ phase. So, it is extremely difficult to obtain single-phase $\mathrm{LaFe}_{12} \mathrm{O}_{19}$

In this study, taking into account the ionic radius and the charge balance of the elements, a new M-type ferrite with substitution of trivalent $\mathrm{La}^{3+}$ and monovalent $\mathrm{K}^{+}$for divalent $\mathrm{Ba}^{2+}, \quad \mathrm{Sr}^{2+}, \mathrm{Pb}^{2+}$ is prepared by a solid-state-reaction. We have investigated the synthesis conditions and the magnetic properties of M-type potassium, lanthanum-iron oxides.

\section{EXPERIMENTAL PROCEDURE}

The samples were prepared by a solid-state-reaction. $\mathrm{K}_{2} \mathrm{CO}_{3}$ (Wako; 99.5\%), $\alpha-\mathrm{Fe}_{2} \mathrm{O}_{3}$ (JFE Chemical; 99.6\%) and $\mathrm{La}_{2} \mathrm{O}_{3}$ (Wako; 99.5\%) reagents were mixed in order to give the composition of $\mathrm{K}_{0.5} \mathrm{La}_{0.5} \mathrm{Fe}_{12} \mathrm{O}_{19}$ by using a 
mortar or a planetary ball mill (Fritsch, P-7 premium line). The mixed powder was pressed into a disk. The disk was put in a platinum crucible, being covered with $\mathrm{KCl}$ powder in order to prevent the evaporation of potassium ions. It was sintered at $1275^{\circ} \mathrm{C}$ in air. The crystal structure was studied with X-ray diffraction (Rigaku RINT Ultima3). The magnetization was measured by using a vibrating sample magnetometer (VSM; Tamakawa TM-VSM2130HGC) and a superconducting quantum interference device magnetometer (SQUID; Quantum Design MPMS-XL). The compositional analysis data of the sample was obtained with an electron probe micro analyzer (EPMA; JEOL JXA-8200).

\section{EXPERIMENTAL RESULT AND DISCUSSION}

Figure 2 shows the X-ray diffraction pattern of the sample with the initial composition at $\mathrm{K}: \mathrm{La}: \mathrm{Fe}=0.5$ : $0.5: 12$. In this $\mathrm{X}$-ray diffraction pattern, diffraction peaks of $\mathrm{KFe}_{11} \mathrm{O}_{17}$ and $\mathrm{M}$-type ferrite, $\alpha-\mathrm{Fe}_{2} \mathrm{O}_{3}$, and $\mathrm{LaFeO}_{3}$ phases were observed. As $\mathrm{KFe}_{11} \mathrm{O}_{17}, \alpha-\mathrm{Fe}_{2} \mathrm{O}_{3}$ and $\mathrm{LaFeO}_{3}$ are nonferromagnetic, they give no contribution to spontaneous magnetization. However, there is a possibility that M-type ferrite is ferromagnetic. In order to investigate this possibility, magnetization of this sample was measured.

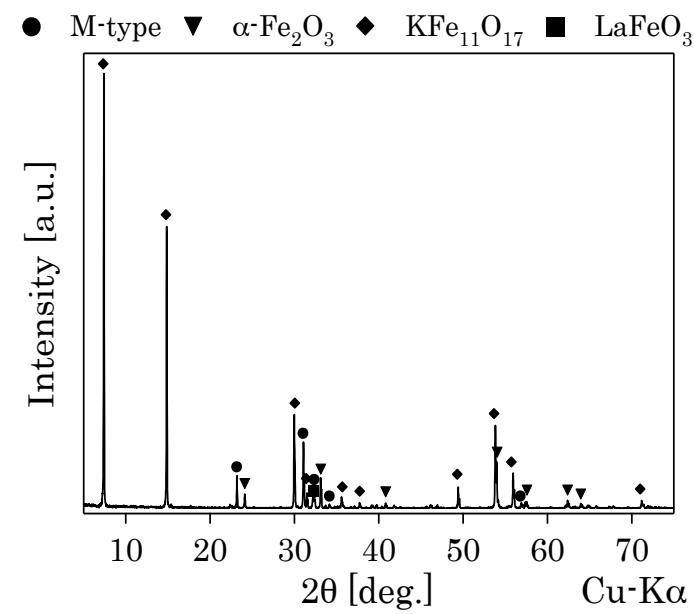

Fig. 2 X-ray diffraction pattern for the sample with the initial composition at $\mathrm{K}: \mathrm{La}: \mathrm{Fe}=0.5: 0.5$ : 12 .

Figure 3 shows the magnetization curve at $300 \mathrm{~K}$ of the sample with the initial composition of $\mathrm{K}: \mathrm{La}: \mathrm{Fe}=0.5$ : $0.5: 12$. The sample is clearly ferromagnetic and the magnetization at $70 \mathrm{kOe}$ is $23.1 \mathrm{emu} / \mathrm{g}$. Although the majority phase is antiferromagnetic $\mathrm{KFe}_{11} \mathrm{O}_{17}$, a small amount of the minority magnetoplumbite phase gives rise to the total magnetization. Therefore, we can expect that the magnetization of this magnetoplumbite phase is comparable with that of the conventional magnetoplumbite $\mathrm{BaFe}_{12} \mathrm{O}_{19}$.

Figure 4 shows the temperature dependence of magnetization at $5 \mathrm{kOe}$ for this ferromagnetic sample. The magnetic transition temperature was $440^{\circ} \mathrm{C}$, which is quite close to that of the magnetoplumbite-type ferrite $\left(\mathrm{T}_{\mathrm{C}}=450^{\circ} \mathrm{C}\right.$ for $\left.\mathrm{BaFe}_{12} \mathrm{O}_{19}\right)$ [13]. No other anomaly was observed in this M-T curve from 50 to $600^{\circ} \mathrm{C} . \alpha-\mathrm{Fe}_{2} \mathrm{O}_{3}$

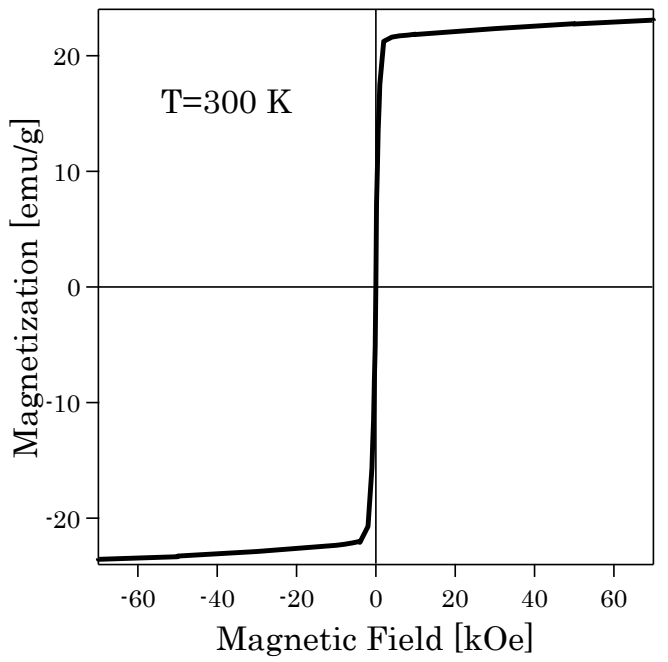

Fig. 3 the magnetization curve at $300 \mathrm{~K}$ of the sample with the initial composition of $\mathrm{K}: \mathrm{La}: \mathrm{Fe}=0.5: 0.5: 12$.

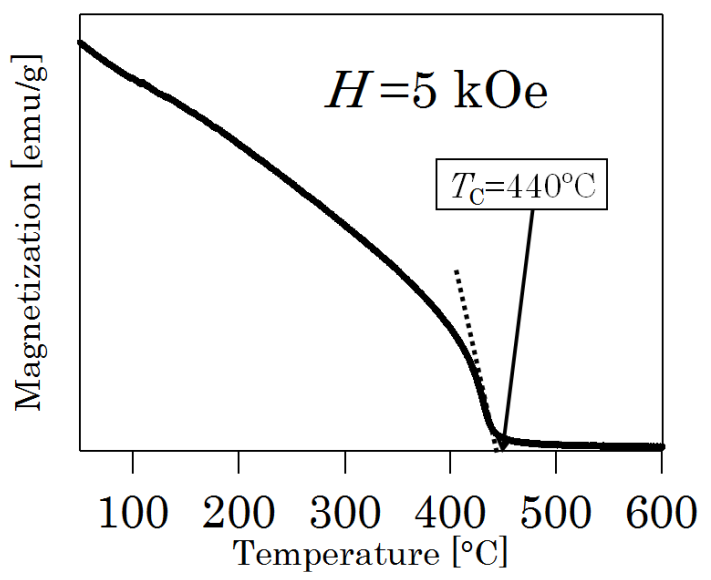

Fig. 4 The temperature dependence of magnetization at 5 $\mathrm{kOe}$ for the sample with the initial composition of $\mathrm{K}: \mathrm{La}$ : $\mathrm{Fe}=0.5: 0.5: 12$.

is a weak ferromagnetic substance originating from Dzyaloshinskii-Moriya interaction. However, this effect did not contribute to the total magnetization since this weak ferromagnet has very weak saturation magnetization of about $1 \mathrm{emu} / \mathrm{g}$ at $\mathrm{RT}$. This result indicates that the magnetism of the sample originates from a new M-type ferrite with lanthanum and potassium ions. Nonetheless, the specimen contains $\mathrm{KFe}_{11} \mathrm{O}_{17}, \alpha-\mathrm{Fe}_{2} \mathrm{O}_{3}$ and $\mathrm{LaFeO}_{3}$, as shown in Fig. 2, which are not ferromagnetic. It is known that $\mathrm{LaFeO}_{3}$ is easily synthesized at $1000^{\circ} \mathrm{C}$ [14]. Most of $\mathrm{K}_{2} \mathrm{CO}_{3}$ reagents seemed to react with $\alpha-\mathrm{Fe}_{2} \mathrm{O}_{3}$ to form $\mathrm{KFe}_{11} \mathrm{O}_{17}$. Thus, unreacted $\alpha-\mathrm{Fe}_{2} \mathrm{O}_{3}$ remained in the product. It should be noted that $\mathrm{La}_{2} \mathrm{O}_{3}$ and $\mathrm{K}_{2} \mathrm{CO}_{3}$ did not react with each other. For this reason, it needs to improve the solid-state-reaction activity of $\alpha-\mathrm{Fe}_{2} \mathrm{O}_{3}$ in order to involve both lanthanum and potassium ions to form a new iron-based oxide. If the surface area of particles is increased, the solid-state-reaction activity will be improved since the reaction paths between 
particles depends on the contact surface area. So, we want to pulverize the starting materials of $\alpha-\mathrm{Fe}_{2} \mathrm{O}_{3}$ and the others more strongly.

Figure 5 shows $\mathrm{x}$-ray diffraction patterns of the sample with the initial composition at $\mathrm{K}: \mathrm{La}: \mathrm{Fe}=0.5: 0.5: 12$ prepared from the starting materials pulverized with the planetary ball mill. The sample prepared from the starting materials milled for $10 \mathrm{~min}$. shows the diffraction peaks of the majority magnetoplumbite phase and the minority $\mathrm{KFe}_{11} \mathrm{O}_{17}$ and $\alpha-\mathrm{Fe}_{2} \mathrm{O}_{3}$ phases. Therefore, the magnetoplumbite phase is effectively synthesized by pulverization of the starting materials. The samples prepared from the starting materials milled for 15 and $30 \mathrm{~min}$. do not show the diffraction peaks of $\mathrm{KFe}_{11} \mathrm{O}_{17}$. The intensity of magnetoplumbite phase is strengthened and that of $\alpha-\mathrm{Fe}_{2} \mathrm{O}_{3}$ phase is decreased with increasing pulverization time of the starting materials. In other words, the volume fraction of magnetoplumbite phase increases and that of $\alpha-\mathrm{Fe}_{2} \mathrm{O}_{3}$ phase reduces with the application of milling to the starting materials.

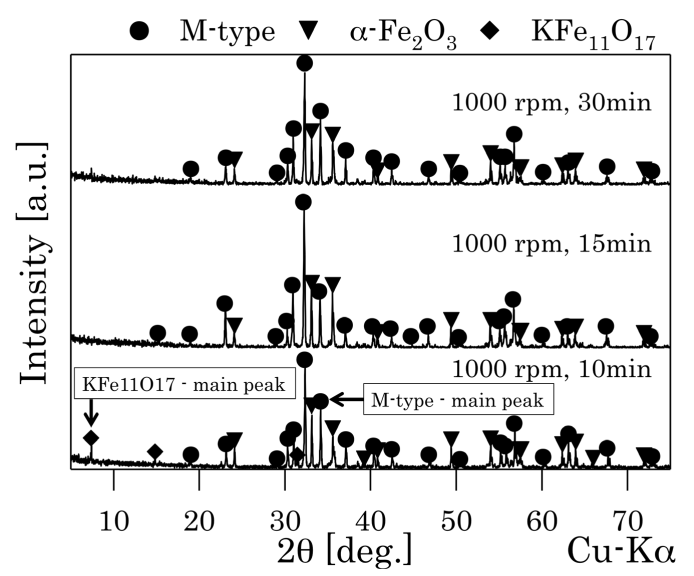

Fig. $5 \mathrm{X}$-ray diffraction patterns of the sample with the composition at $\mathrm{K}: \mathrm{La}: \mathrm{Fe}=0.5: 0.5: 12$ prepared from the starting materials pulverized with a planetary ball mill.

Table 1 shows the magnetizations of the samples prepared from different starting materials. The saturation magnetization increases with decreasing the amounts of $\mathrm{KFe}_{11} \mathrm{O}_{17}$ and $\alpha-\mathrm{Fe}_{2} \mathrm{O}_{3}$ phases, which indicates that the ferromagnetic property is caused by the magnetoplumbite phase.

Table. 1. The magnetizations of the samples prepared from different starting materials.

\begin{tabular}{|c|c|}
\hline Milling process & Magnetization [emu/g] \\
\hline Mortar & 23.1 \\
\hline $1000 \mathrm{rpm}, 10 \mathrm{~min}$ & 54.5 \\
\hline $1000 \mathrm{rpm}, 15 \mathrm{~min}$ & 59.6 \\
\hline $1000 \mathrm{rpm}, 30 \mathrm{~min}$ & 60.5 \\
\hline
\end{tabular}

Figure 6 shows a SEM photo of the sample prepared from the starting materials milled for $15 \mathrm{~min}$. The SEM result clearly indicates the existence of two different particles.We set the measuring point $\mathrm{A}$ on a typical hexagonal particle, and the measuring point $\mathrm{B}$ on a relatively small spherical particle. The chemical compositions at the measuring points $\mathrm{A}$ and $\mathrm{B}$ were studied by EPMA. The obtained EPMA compositional analysis data of measuring point $\mathrm{A}$ are $\mathrm{K}: \mathrm{La}: \mathrm{Fe}=0.75$ : $0.90: 18.58$. The measuring point $\mathrm{B}$ only contains iron and oxygen, so this particle seems to be $\alpha-\mathrm{Fe}_{2} \mathrm{O}_{3}$. From these results, the composition of the magnetoplumbite phase in this sample is estimated to be $\mathrm{K}_{0.45} \mathrm{La}_{0.55} \mathrm{Fe}_{12} \mathrm{O}_{19-\diamond \text {. }}$

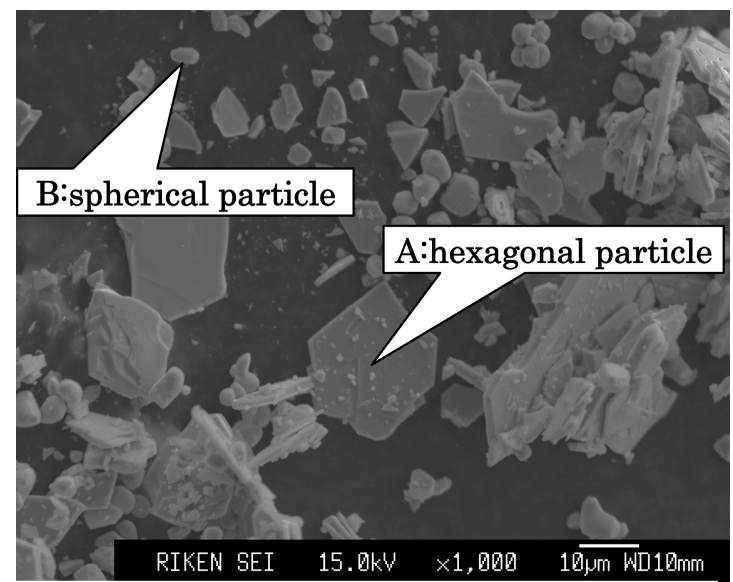

Fig. 6 SEM photo of the sample prepared from the starting materials milled for $15 \mathrm{~min}$.

\section{SUMMARY}

We have investigated the synthesis conditions, and the magnetic properties of M-type ferromagnetic potassium, lanthanum-iron oxides. The samples were prepared by a solid-state-reaction. $\mathrm{K}_{2} \mathrm{CO}_{3}, \alpha-\mathrm{Fe}_{2} \mathrm{O}_{3}$ and $\mathrm{La}_{2} \mathrm{O}_{3}$ reagents were mixed in order to give the composition of $\mathrm{K}_{0.5} \mathrm{La}_{0.5} \mathrm{Fe}_{12} \mathrm{O}_{19}$ by using a mortar or a planetary ball mill. In the X-ray diffraction pattern of the sample prepared from the mortar-mixed starting materials with the initial composition at $\mathrm{K}: \mathrm{La}: \mathrm{Fe}=0.5: 0.5: 12$, diffraction peaks of $\mathrm{KFe}_{11} \mathrm{O}_{17}$ and M-type, $\alpha-\mathrm{Fe}_{2} \mathrm{O}_{3}$, and $\mathrm{LaFeO}_{3}$ phases are observed. From the magnetization curve at $300 \mathrm{~K}$ of the sample, the specimen is clearly ferromagnetic and the magnetization at $70 \mathrm{kOe}$ was 23.1 $\mathrm{emu} / \mathrm{g}$. The magnetic transition temperature of the sample is $440^{\circ} \mathrm{C}$ which is quite close to that of the magnetoplumbite-type ferrite. No other anomaly is observed in the M-T curve from 50 to $600^{\circ} \mathrm{C}$. This result indicates that the magnetism of the sample originates from a new M-type ferrite with lanthanum and potassium ions. Next, we pulverized the starting materials of $\alpha-\mathrm{Fe}_{2} \mathrm{O}_{3}$ and the others more strongly. The sample prepared from the starting materials milled for 10,15 and $30 \mathrm{~min}$. shows the diffraction peaks of the majority magnetoplumbite phase. The saturation magnetization increases with decreasing the amount of $\mathrm{KFe}_{11} \mathrm{O}_{17}$ and $\alpha-\mathrm{Fe}_{2} \mathrm{O}_{3}$ phases, which indicates that the ferromagnetic property is caused by the magnetoplumbite phase. The obtained EPMA compositional analysis data of a hexagonal particle are 
$\mathrm{K}: \mathrm{La}: \mathrm{Fe}=0.75: 0.90: 18.58$. From this result, the composition of the magnetoplumbite phase in this sample is estimated to be $\mathrm{K}_{0.45} \mathrm{La}_{0.55} \mathrm{Fe}_{12} \mathrm{O}_{19-\text { s。 }}$.

Acknowledgment

We are most grateful to Mr. Kowashi Watanabe for his permission to use the EPMA apparatus and his help in the measurement.

\section{References}

[1] H. Kojima, in: E. P. Wolfarth (Ed.), Ferromagnetic Materials, vol. 3, North-Holland, Amsterdam, 1982.

[2] L. Pust, P.E. Wigen, M. Ramesh, P. Siroky, K. Suk, J. Magn. Magn. Mater. 54-7 (1986) 1195

[3] A.C. Stergiou, D. Samaras, O. Kalogirou, H. Vincent, A. Bekka, Solid State Ion. 50 (1992) 11

[4] R. Sole, X. Ruiz, R. Cabre, M. Aguilo, F. Díaz, V. Nikolov, J. Crysral Growth. 166 (1996) 402

[5] F. L. Wei, H. C. Fang, C. K. Ong, C. S. Wang, Z. Yang, J. Appl. Phys. 87 (2000) 8636

[6] M. W. Pieper, F. Kools, A. Morel, Phys. Rev. B 65 184402 (2002)

[7] P. Novak, K. Knizek, M. Kupferling, R. Grossinger, M. W. Pieper, Eur. Phys. J. B. 43 (2005) 509

[8] X. Liu, W. Zhang, Sen Yang, Zhi Yu, Benxi Gu, Youwei Du, J. Magn. Magn. Mater. 238 (2002) 207

[9] G. Wiesinger, M. Muller, R. Grossinger, M. Pieper, A. Morel, F. Kools, P. Tenaud, J. M. Le Breton, J. Kreisel, Phys. Stat. Sol. 189 (2002) 499

[10] F. K. Lotgeting, J. Phys. Chem. Solids 35 (1974) 1633

[11] J. Smit and H. P. Wijn, Ferrites (Wiley, New York, 1959)

[12] M. Kupferling, V.Corral Flores, R. Grossinger, J. Matutes Aquino, J. Magn. Magn. Mater. 290-291 (2005) 1255

[13] Belov, K. P., L. I. Korovela, R. Z. Levitin, Yu. V. Jergin, A. V. Pedko, Phys. Stat. Sol. 12 (1965) 219

[14] M. Takano, J. Kawachi, N. Nakanishi, Y. Takeda, J. Solid State Chem., 39 (1981) 75

(Received December 28, 2011; Accepted May 9, 2012) 